\title{
Downregulation of IncRNA RPLP0P2 inhibits cell proliferation, invasion and migration, and promotes apoptosis in colorectal cancer
}

\author{
HANG YUAN ${ }^{1,2}$, SHILIANG TU ${ }^{2}$, YINGYU MA ${ }^{3}$ and YUEMING SUN ${ }^{1}$ \\ ${ }^{1}$ Department of General Surgery, The First Affiliated Hospital of Nanjing Medical University, Nanjing, \\ Jiangsu 210000; ${ }^{2}$ The Surgical Department of Coloproctology; ${ }^{3}$ Clinical Research Institute, Zhejiang Provincial \\ People's Hospital, People's Hospital of Hangzhou Medical College, Hangzhou, Zhejiang 310014, P.R. China
}

Received July 19, 2020; Accepted December 31, 2020

DOI: $10.3892 / \mathrm{mmr} .2021 .11948$

\begin{abstract}
Recent studies have revealed that long noncoding RNAs (lncRNAs) are closely associated with colorectal cancer (CRC); however, the role of the IncRNA RPLP0P2 in CRC remains largely unknown. In the present study, RNA expression profiles of CRC were collected from The Cancer Genome Atlas database and the prognosis of CRC with respect to RPLP0P2 was assessed. Subsequently, RPLP0P2 expression was knocked down in the human CRC cell line RKO using a short hairpin RNA (shRNA) lentivirus, and the biological behaviors of the cells, such as proliferation, migration, cell cycle progression and apoptosis, were examined. The results demonstrated that the expression levels of RPLP0P2 were higher in CRC tissue compared with those in normal tissue, and RPLP0P2 was associated with prognosis. RPLP0P2 knockdown significantly decreased cell colony formation, migration and invasion, and arrested CRC cells in the $S$ phase to $G_{2} / M$ phase transition. Furthermore, apoptosis was significantly increased in CRC cells infected with the RPLP0P2 shRNA lentivirus compared with in the control group. In conclusion, RPLP0P2 may promote proliferation, invasion and migration, and inhibit apoptosis of CRC cells, suggesting that RPLP0P2 may function as an oncogene in $\mathrm{CRC}$.
\end{abstract}

\section{Introduction}

Colorectal cancer (CRC) is a common type of malignant tumor and a frequent cause of cancer-related death worldwide (1). Notably, CRC is the fourth most frequent gastroenteric tumor and is associated with high morbidity in

Correspondence to: Professor Yueming Sun, Department of General Surgery, The First Affiliated Hospital of Nanjing Medical University, 300 Guangzhou Road, Nanjing, Jiangsu 210000, P.R. China

E-mail: jssym@vip.sina.com

Key words: RPLP0P2, colorectal cancer, proliferation, apoptosis
China (2). Although encouraging progress for early diagnosis and treatment of CRC has been achieved in recent decades, the prognosis and 5-year survival rate of patients with CRC is still unsatisfactory (3). The tumorigenesis and progression of $\mathrm{CRC}$ is a complex pathological process involving numerous molecules and pathways, and the underlying mechanisms have yet to be fully elucidated. Therefore, further insight into the molecular mechanisms of CRC may help improve the diagnosis and prognosis, and consequently identify novel therapeutic targets for CRC.

Noncoding RNAs (ncRNAs) have been reported to serve important biological roles in transcriptional regulation, RNA modification, formation of chromosomes and cellular development (4). Notably, ncRNAs are further grouped into small ncRNAs $(<200 \mathrm{bp})$ and long ncRNAs (lncRNAs; $>200 \mathrm{bp}$, up to $100 \mathrm{~kb}$ ) based on transcript size, and lncRNAs expression levels are indicative of those of active molecules and lack significant open reading frames $(5,6)$. Previous studies have demonstrated that lncRNAs may serve important roles in several biological processes, including cell proliferation, differentiation and apoptosis $(7,8)$. In addition, it has been reported that dysregulation of lncRNAs may be involved in the development of human cancer (9-15). The IncRNA RPLP0P2 has been reported to exhibit significantly decreased expression in lung adenocarcinoma (16). Chen et al (17) also observed that RPLP0P2 expression was lower in lung adenocarcinoma, and low expression of RPLP0P2 was associated with poor prognosis, as well as decreased proliferation and adhesive ability, in tumor cells. Conversely, Li et al (18) revealed that RPLP0P2 was negatively associated with overall survival in gastric cancer, suggesting that it may act as an oncogene in gastric cancer. These studies indicated that the role of RPLPOP2 in cancer is related to its tissue specificity.

Zhong et al (19) performed bioinformatics analysis on RPLP0P2 in CRC and revealed that RPLP0P2 may represent an important oncogene, which was negatively associated with overall survival. This finding suggests that RPLP0P2 may have an important role in CRC; however, the specific biological role and mechanism of RPLPOP2 remain unclear. The present study demonstrated that the IncRNA RPLPOP2 may act as a candidate CRC biomarker according to lncRNA 
gene expression data from The Cancer Genome Atlas (TCGA) RNA-sequencing (RNA-Seq) datasets. These findings demonstrated that the IncRNA RPLP0P2 may have an important role in CRC; therefore, the present study aimed to further determine the biological role and clinical significance of RPLPOP2 in $\mathrm{CRC}$ and to investigate the underlying molecular mechanisms.

\section{Materials and methods}

Datasets and TCGA bioinformatics. TCGA data portal (https://tcga-data.nci.nih.gov/docs/publi cations/tcga/) was used to locate microarray studies focusing on expression of RPLPOP2 in CRC, and 50 matched cases of CRC (27 females and 23 males; age, 40-90 years; mean age $70.34 \pm 13.36$ years) and paired adjacent normal tissue, along with 647 cases of CRC (294 females and 353 males; age, 31-90 years; mean age, $66.17 \pm 12.78$ years) and 51 unpaired cases of normal tissue were collected, which were used for RPLP0P2 expression analysis. The criterion for dividing the expression of RPLPOP2 into high and low was the median value of gene expression. Furthermore, 308 CRC cases (138 females and 170 males; age, 31-90 years; mean age, $67.29 \pm 12.79$ years old) were collected for survival analysis.

Cell culture. The human CRC cell lines (HCT116, HT29, SW480 and RKO) were purchased from The Cell Bank of Type Culture Collection of The Chinese Academy of Sciences. Cells were cultured in RPMI-1640 medium supplemented with $10 \%$ fetal bovine serum (Biological Industries) and were maintained at $37^{\circ} \mathrm{C}$ in an atmosphere containing $5 \% \mathrm{CO}_{2}$. Digestion and passage of cells were performed until they reached $80 \%$ confluence.

Reverse transcription-quantitative PCR (RT-qPCR). Total RNA was extracted from CRC cells using Total RNA Isolation Reagent (cat. no. 3101-100; Shanghai Pufei Biotech Co., Ltd.) in accordance with the manufacturer's instructions. The M-MLV (Moloney Murine Leukemia Virus Reverse Transcriptase) kit (cat. no. M1705; Promega Corporation) was used to reverse transcribe total RNA according to the manufacturer's protocol. The expression levels of RPLPOP2 and GAPDH were assessed in CRC cells by qPCR using SYBR Master Mixture (cat. no. DRR041B; Takara Bio, Inc.). The thermocycling conditions were as follows: $95^{\circ} \mathrm{C}$ for $30 \mathrm{sec}, 95^{\circ} \mathrm{C}$ for $5 \mathrm{sec}$ and $60^{\circ} \mathrm{C}$ for $30 \mathrm{sec}, 40$ cycles. Primer sequences were as follows: RPLP0P2, forward 5'-TCAAGACGGGAG ACAAAGTGG-3' and reverse 5'-TCGATGATAGAATGGGGCACT-3' (247 bp product); GAPDH, forward 5'-TGACTTCAACAGCGACACCCA-3' and reverse 5'-CACCCTGTTGCTGTAGCCAAA-3' (121 bp product). All samples were normalized to GAPDH, and all experiments were performed in triplicate.

The mean value of each triplicate was used to calculate relative lncRNA expression levels. $2^{-\Delta \Delta C q}$ method was used to detect RNA level (20).

Lentivirus-mediated RPLPOP2 short hairpin RNA (shRNA) vector infection. A shRNA lentivirus targeting RPLP0P2 was constructed using the GV248 (hU6-MCS-Ubiquitin-EGFP-IRES-puromycin) vector (Shanghai GeneChem Co., Ltd.). The shRNAs were synthesized and inserted into GV248 lentivirus vector, and then verified by DNA sequencing (Sanger). The shRNA sequences were as follows: shRNA-1, forward 5'-CCGGCC CTGTAAAGTACCTGGAATACTCGAGTATTCCAG GTA CTTTACAGGGTTTTTG-3' and reverse 5'-AATTCAAAA ACCCTGTAAAGTACCTGGA ATACTCGAGTATTCC AGGTACTTTACAGGG-3'; shRNA-2, forward 5'-CCGGCT GATCAAGACGGGAGACAAACTCGAGTTTGTCTC CCG TCTTGATCAGTTTTTG-3' and reverse 5'-AATTCAAAA ACTGATCAAGACGGGAGACAAACTCGAGTTTGT CTC CCGTCTTGATCAG-3'; shRNA-3, forward 5'-CCGGGTCAG ACGAGGATATGGGATTCTCGAGAATCCCATATCCTCG TCTGACTTTTTG-3' and reverse 5'-AATTCAAAAAGTC AGACGAGGATATGGGATTCTCGAGAATCCCATA TCC TCGTCTGAC-3'. Lower case indicates protective bases; upper case indicates the primer sequence.

As a negative control, a shRNA with a scrambled sequence (TTCTCCGAACGTGTCACGT) was used. Subsequently, the lentivirus-mediated RPLPOP2 shRNA vector was confirmed via sequencing and used for subsequent experiments.

For infection, $1 \times 10^{5}$ RKO cells/per well were seeded into 6-well plates and were incubated for $24 \mathrm{~h}$ at $37^{\circ} \mathrm{C}$, after which, medium containing the lentivirus (multiplicity of infection, 10) was added, according to the manufacturer's instructions. RKO cells were infected with the lentivirus for $72 \mathrm{~h}$ at $37^{\circ} \mathrm{C}$ and infection efficiency was assessed. The approximate fluorescence infection efficiency was estimated by comparing bright-field and fluorescent images in the same field. Subsequently, RT-qPCR was performed to detect knockdown efficiency.

Detection of cell proliferation by Cell Counting Kit-8 (CCK-8) and colony formation assay. RKO cells were infected with the RPLPOP2 shRNA lentivirus for 3 days, and were then trypsinized and resuspended in medium. For the CCK- 8 assay, $2 \times 10^{4}$ cells/well were seeded into 96-well plates. After $24 \mathrm{~h}$, $10 \mu$ l CCK-8 solution (Beyotime Institute of Biotechnology) was added to each well at $0,1,2$ and 3 days, and the cells were incubated at $37^{\circ} \mathrm{C}$ for $2 \mathrm{~h}$. The absorbance at each time point was measured at $450 \mathrm{~nm}$ using a microplate reader.

For the colony formation assay, $2 \mathrm{ml}$ medium, including RKO cells (500 cells), was added into each well of 6-well culture plates, and three replicates for each group were performed. Cells were further cultured at $37^{\circ} \mathrm{C}$ in an atmosphere containing $5 \% \mathrm{CO}_{2}$ for 8 days; the medium was changed every 3 days while cell state was observed. After 8 days, a fluorescence microscope was used to image the cell colonies. Briefly, $1 \mathrm{ml} 4 \%$ paraformaldehyde was used to fix the cells for 30-60 min at room temperature and $1 \mathrm{ml}$ crystal violet staining solution was added to stain the cells for 10-20 min at room temperature. The cells were then washed several times and air dried, and the number of colonies containing $>50$ cells was counted.

Detection of cell migration by Celigo imaging cytometry. Cells were seeded into 96-well plates at a concentration of $50,000$ cells/well (100 $\mu \mathrm{l} / \mathrm{well})$ and grown to $90 \%$ confluence. Each group had three replicates and the cells were cultured at $37^{\circ} \mathrm{C}$ in an atmosphere containing $5 \% \mathrm{CO}_{2}$ for $24 \mathrm{~h}$. Subsequently, the Celigo scratch instrument was used to gently 
push upward to form scratches in the cell layer. Celigo scanning (cat. no. VP408FH; V\&P Scientific, Inc.) was performed at 24 and $48 \mathrm{~h}$ according to the degree of healing, and the migration rate was calculated. Celigo was used to to obtain the cell area (S), migration area was calculated by $\mathrm{S}_{\mathrm{Xh}}-\mathrm{S}_{0 \mathrm{~h}}$ $(\mathrm{X}=24$ or 48$)$, and migration rate was calculated by $\left(\mathrm{S}_{\mathrm{Xh}}-\mathrm{S}_{0 \mathrm{~h}}\right) /$ area of scratch.

Cell migration and invasion assays. A 24-well plate containing pore inserts (size, $8 \mu \mathrm{m}$ ) with or without Matrigel-coated filters (Corning, Inc.) was used for migration and invasion assay. In the upper chamber, $500 \mu 1$ cell suspension in serum-free medium $\left(10^{5}\right.$ cells/well) was added, and $750 \mu \mathrm{l}$ medium containing $10 \%$ FBS was added into the lower chamber; the cells were cultured at $37^{\circ} \mathrm{C}$ in an atmosphere containing $5 \%$ $\mathrm{CO}_{2}$ for $72 \mathrm{~h}$. Subsequently, the migratory or invaded cells were stained with Giemsa for 3-5 min and air dried. Migratory or invasive cells per field were imaged under a light microscope and invasion rate was calculated. The number of cells $(\mathrm{N})$ in 5 fields was counted under the microscope, and the control group was used as reference. The invasion rate was calculated as $\mathrm{N}_{\text {experimental group }} / \mathrm{N}_{\text {control group }}$.

Cell cycle assay. Cells were cultured in $6-\mathrm{cm}$ dishes to $\sim 80 \%$ confluence and were digested and resuspended. After centrifugation $\left(400 \mathrm{x} \mathrm{g}, 10 \mathrm{~min}\right.$ at $\left.4^{\circ} \mathrm{C}\right)$, cells were washed with D-Hanks (pH, 7.2-7.4) and fixed in 75\% ethanol for $1 \mathrm{~h}$ at $4^{\circ} \mathrm{C}$. Cells were stained with cell cycle dye, which was prepared using 40X propidium iodide ( $2 \mathrm{mg} / \mathrm{ml}$; cat. no. $\mathrm{P} 4170$; Sigma-Aldrich; Merck KGaA), 100X RNase (10 mg/ml; cat. no. EN0531; Fermentas; Thermo Fisher Scientific, Inc.) and 1X D-Hanks at a ratio of 25:10:1,000. After incubation for $30 \mathrm{~min}$ at room temperature in the dark, samples were analyzed with a flow cytometer (Guava easyCyte HT; EMD Millipore) to detect DNA content. Guava InCyte software (3.1.1.0; EMD Millipore) was used for analysis.

Apoptosis assay. Cells $\left(5 \times 10^{5}\right)$ were trypsinized, collected and centrifuged at $400 \times \mathrm{g}$ for $5 \mathrm{~min}$ at $4^{\circ} \mathrm{C}$. Subsequently, pre-cooled D-Hanks ( $\mathrm{pH}, 7.2-7.4$ ) was used to wash the cells, and they were resuspended in $200 \mu \mathrm{l} 1 \mathrm{X}$ binding buffer (eBioscience; Thermo Fisher Scientific, Inc.) and stained with $10 \mu \mathrm{l}$ Annexin V-APC (eBioscience; Thermo Fisher Scientific, Inc.) in the dark at room temperature for 10-15 min. According to the number of cells, 400-800 $\mu$ l binding buffer was added and samples were assessed using a flow cytometry analyzer (Guava easyCyte HT; EMD Millipore). Guava InCyte software (3.1.1.0; Millipore) was used for analysis.

Statistical analysis. Data are presented as the mean \pm SD $(n=3)$. All results were analyzed using SPSS 16.0 (SPSS, Inc.). The expression levels of RPLP0P2 in normal and cancer tissues were compared using the Mann-Whitney $U$ test and Wilcoxon signed-rank test. Survival analysis with regard to RPLP0P2 expression levels in patients with CRC was estimated by the Kaplan-Meier method accompanied by the log-rank test to calculate differences between the curves. Comparisons between experimental and control groups in colony formation, wound-healing assay, Transwell assay, cell cycle progression analysis and apoptosis analysis were performed by paired-samples t-test. Differences in the effect of RPLP0P2 shRNA on knockdown were compared using one-way ANOVA and Dunnett's test was used for intra-group comparison. $\mathrm{P}<0.05$ was considered to indicate a statistically significant difference.

\section{Results}

Expression levels of RPLPOP2 are high in CRC and are associated with prognosis. The present study analyzed the expression levels of RPLP0P2 from lncRNA gene expression data in TCGA RNA-Seq datasets and revealed that the expression levels of RPLPOP2 were higher in CRC tissues compared with those in normal tissues (Fig. 1A). Paired samples of CRC also revealed that RPLPOP2 expression levels were higher in CRC tissue compared with paracancerous normal tissue (Fig. 1B). Further analysis revealed that high levels of RPLP0P2 were associated with poor survival in patients with CRC (Fig. 1C).

Infection efficiency of the RPLPOP2 shRNA lentivirus in $R K O$ cells. In order to verify expression levels of RPLPOP 2 in CRC, levels of RPLP0P2 in CRC cell lines were assessed by RT-qPCR; results showed that RPLP0P2 levels were higher in RKO cells compared with other CRC cells (HCT116, HT29 and SW480; Fig. 1D). Subsequently, RKO cells were used to assess the biological role of RPLP0P2 using a shRNA lentivirus.

RKO CRC cells were infected with the RPLP0P2 shRNA lentivirus for $72 \mathrm{~h}$ and then observed using a fluorescence microscope. The results revealed that the cells appeared normal after infection, and the infection efficiency of the shRNA lentivirus was $\sim 80 \%$ (Fig. 2A). RT-qPCR also revealed that the expression levels of RPLP0P2 were significantly downregulated by the RPLP0P2 shRNA lentivirus in all groups, particularly in the shRNA-3 group (Fig. 2B). Therefore, RPLP0P2 shRNA-3 was used in subsequent experiments. CCK-8 cell proliferation assay revealed that shRNA-RPLP0P2 knockdown significantly decreased proliferation in RKO cells (Fig. 2C).

RPLPOP2 shRNA lentivirus suppresses colony formation in $R K O$ cells. Following infection with the RPLPOP2 shRNA lentivirus, the colony-forming ability of RKO cells was assessed. The results revealed that RPLP0P2 knockdown significantly decreased the number of cell colonies, whereas the control group exhibited a normal ability to pack together and form colonies (Fig. 3), suggesting that RPLP0P2 may be associated with the clonogenic capacity of RKO cells.

RPLPOP2 shRNA lentivirus suppresses migration and invasion of RKO cells. The effects of RPLPOP2 on RKO cell migration and invasion were assessed by wound-healing and Transwell assays. The results of the wound-healing assay revealed that the wound area of RPLPOP2 shRNA-infected cells was significantly larger compared with that in the control group (Fig. 4A), indicating that RPLPOP2 shRNA inhibited cell migration. The results of the Transwell assay demonstrated that the number of migrated cells and invaded cells in the RPLPOP2 shRNA-infected group was significantly decreased compared with in the control group (Fig. 4B). 

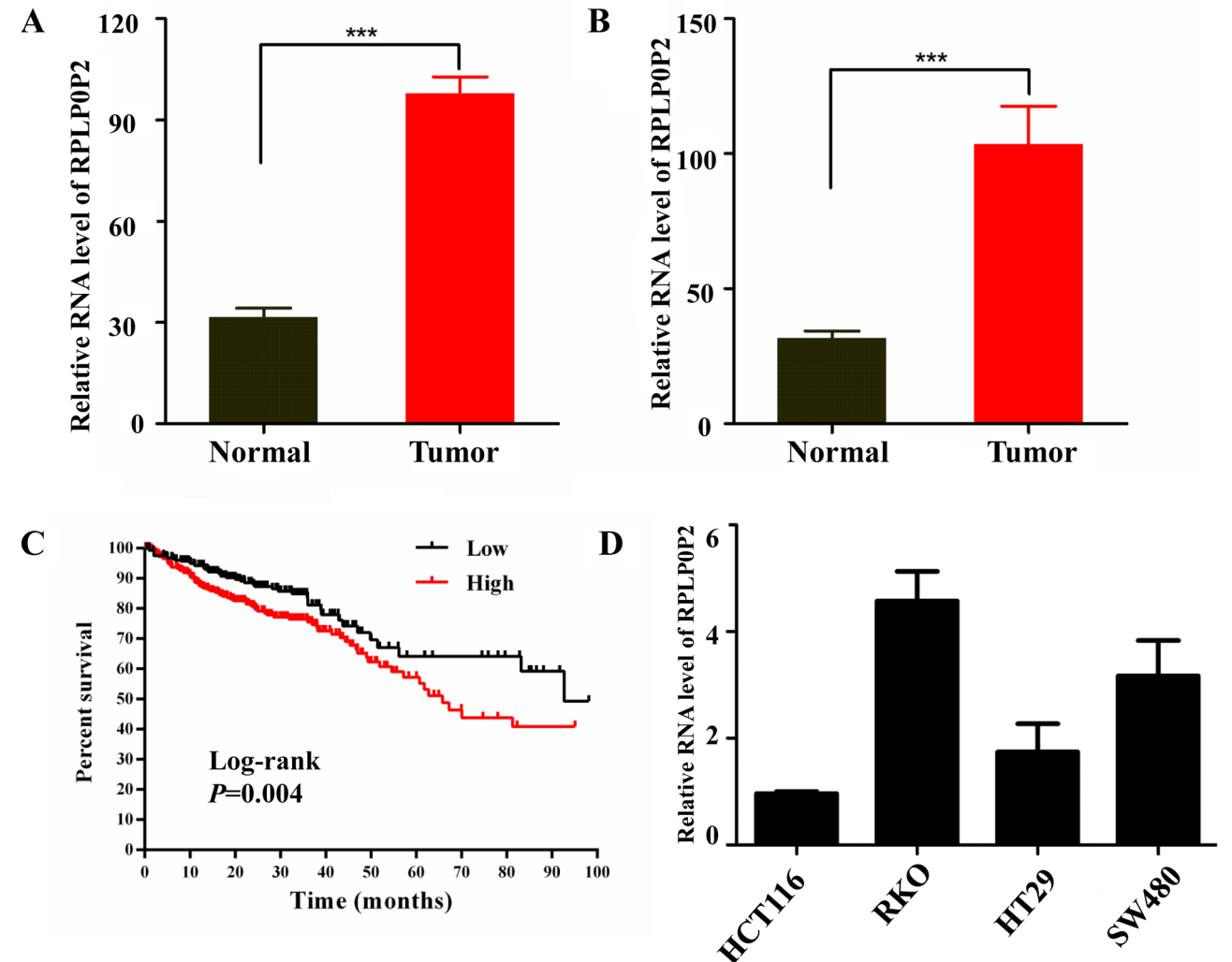

Figure 1. Long non-coding RNA RPLPOP2 levels are elevated in CRC tissues. RPLPOP2 expression data in CRC tissues were obtained from The Cancer Genome Atlas database. (A) RPLP0P2 expression levels were higher in CRC tissues compared with in normal tissues (analysis of 51 cases of normal tissue and 647 cases of CRC tissue; test of two independent samples (Mann Whitney U test). (B) RPLPOP2 expression levels in 50 cases of matched CRC and normal tissue samples (test of paired samples, Wilcoxon signed-rank test). (C) High RPLPOP2 levels are associated with poor prognosis in patients with CRC. (D) Relative expression levels of RPLP0P2 in CRC cell lines. ${ }^{* * *} \mathrm{P}<0.001$. CRC, colorectal cancer.

A

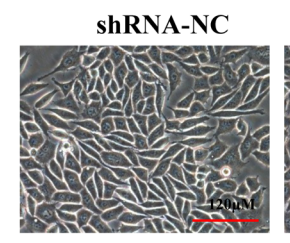

ShRNA-1

ShRNA-2

ShRNA-3
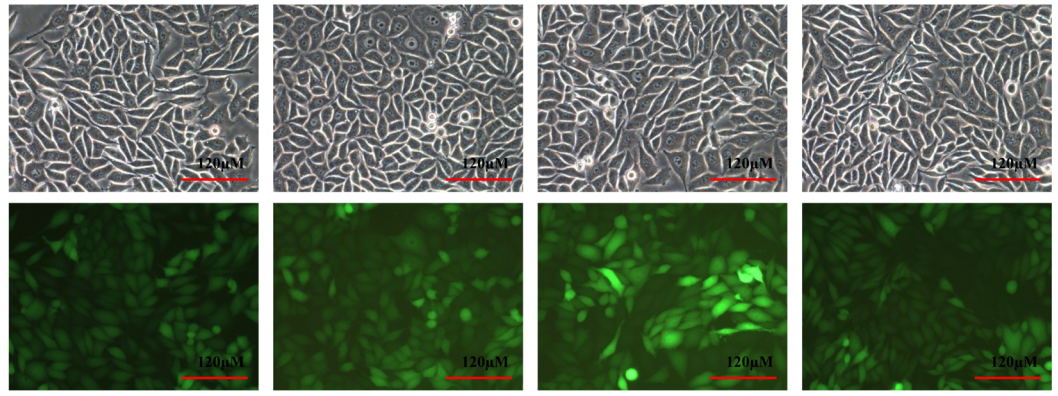

B

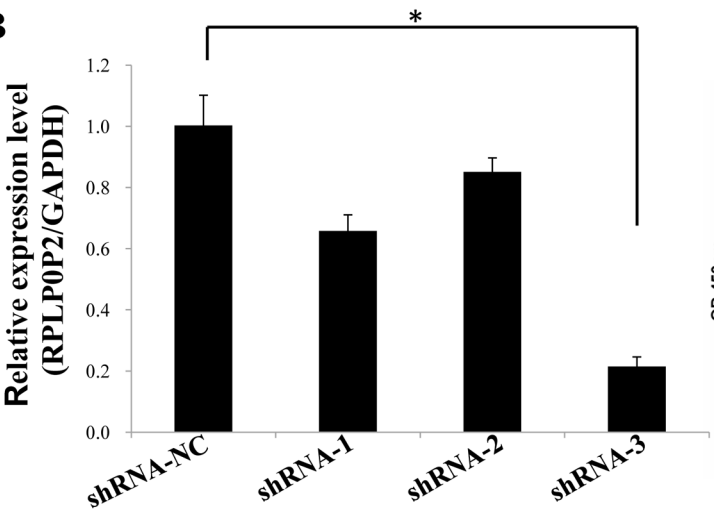

C

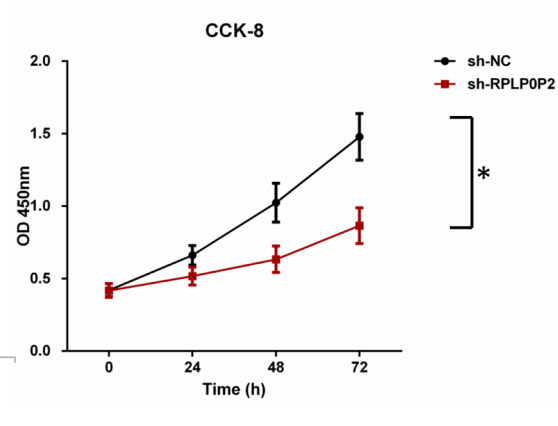

Figure 2. Knockdown efficiency of RPLP0P2 with shRNA lentivirus in RKO cells. (A) Transfection efficiency of the RPLP0P2 shRNA lentivirus in RKO cells was $\sim 80 \%$. Upper rows, bright-field images of lentivirus infection; lower rows, fluorescent images of lentivirus infection. (B) Relative expression levels of RPLPOP2 were detected and it was revealed that the knockdown efficiency of shRNA-3 was the best. (C) CCK-8 cell proliferation assay revealed that shRNA-RPLP0P2 knockdown significantly decreased proliferation of RKO cells. "P<0.05. shRNA, short hairpin RNA; NC, negative control; CCK-8, Cell Counting Kit-8; OD, optical density. 


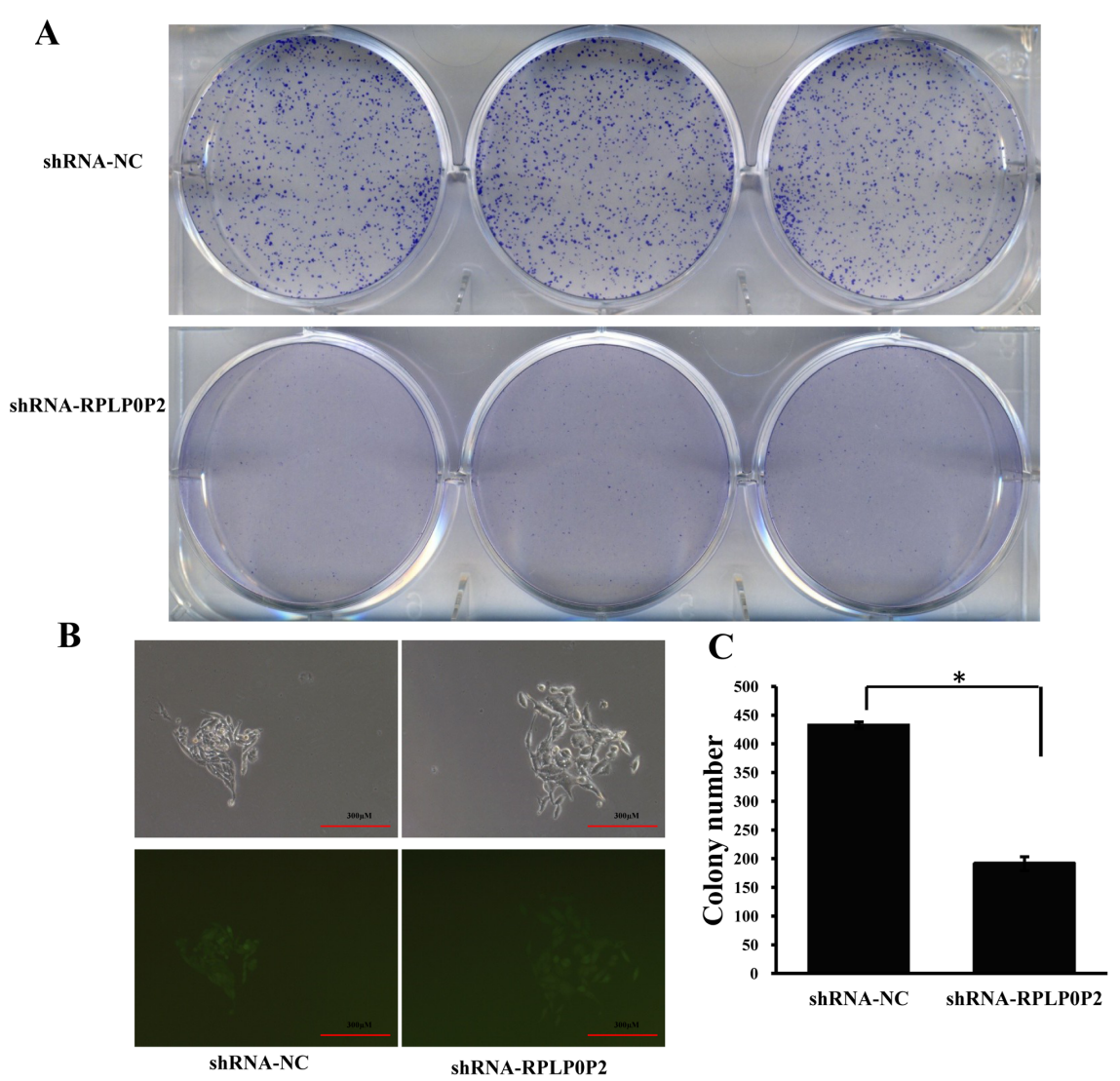

Figure 3. RPLPOP2 knockdown inhibits cell colony formation. (A) Colony formation of RKO cells, which were stained with crystal violet. (B) Colony formation observed microscopically. Upper rows, bright field; lower rows: fluorescent view. (C) Number of stained colonies in RKO cells following infection with the RPLPOP2 shRNA lentivirus. Data are presented as the mean $\pm \mathrm{SD}$. ${ }^{*} \mathrm{P}<0.05$. shRNA, short hairpin RNA; NC, negative control.

A
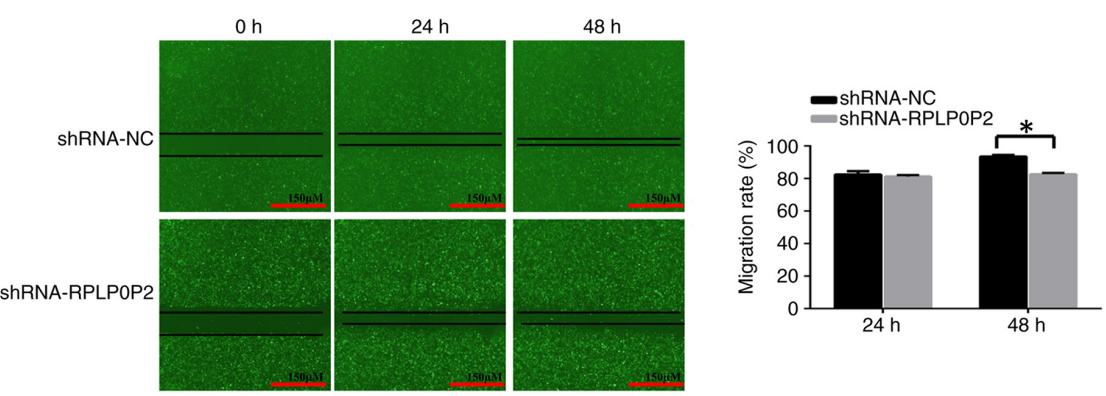

B
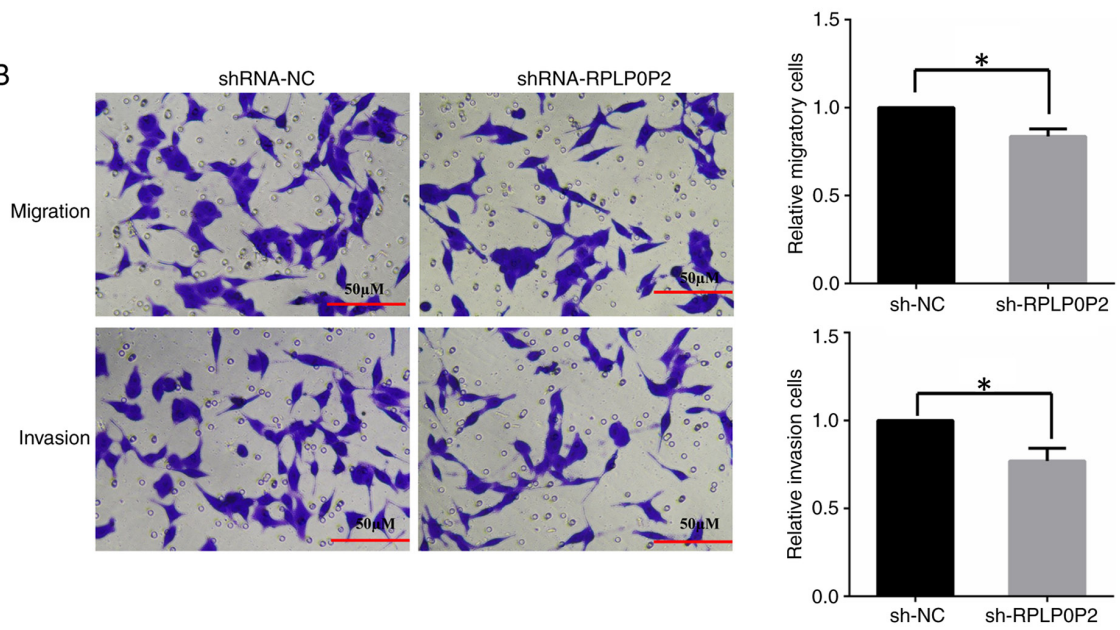

Figure 4. RPLP0P2 knockdown inhibits cell migration and invasion. (A) Wound area of the shRNA RPLP0P2 group was larger than in the control group after $48 \mathrm{~h}$. Representative images and statistical analysis of the wound-healing assay are shown. Magnification, x200. (B) Migration and invasion were reduced in the shRNA RPLP0P2 group compared with in the control group. Representative images of Transwell assay are shown. Magnification, $\mathrm{x} 200$. " $\mathrm{P}<0.05$. shRNA/sh, short hairpin RNA; NC, negative control. 

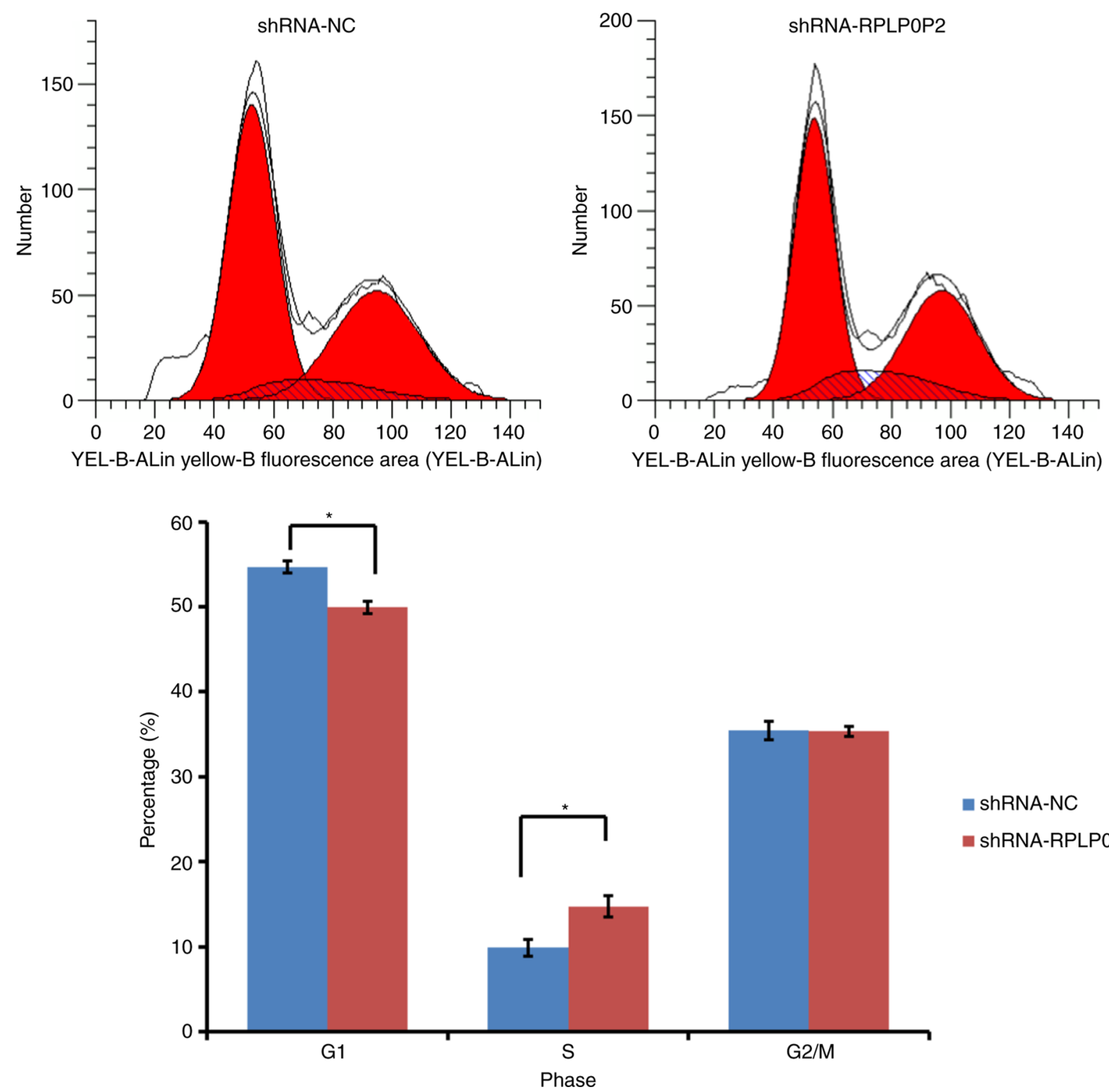

- ShRNA-NC

a shRNA-RPLPOP2

Figure 5. Flow cytometric analysis of cell cycle progression. RPLPOP2 shRNA lentivirus enhanced the proportion of RKO cells in S phase and reduced the proportion of cells in $\mathrm{G}_{1}$ phase. ${ }^{*} \mathrm{P}<0.05$. shRNA, short hairpin RNA; NC, negative control.

RPLPOP2 shRNA arrests RKO cells at S stage. The present study also assessed changes in cell cycle progression in response to RPLP0P2 knockdown with flow cytometry. The results detected increased accumulation of RKO cells in $\mathrm{S}$ phase in the RPLP0P2 knockdown group compared with in the control group, which was evidenced by a significant increase in the number of S phase cells (Fig. 5). Consistently, this result was accompanied by a significant reduction in the percentage of cells in $G_{1}$ phase. Meanwhile, there was no significant difference in $\mathrm{G}_{2} / \mathrm{M}$ cell populations between the RPLP0P2 knockdown and control groups. These results revealed that shRNA-mediated RPLP0P2 knockdown arrested RKO cells from $S$ stage to $G_{2} / M$ stage.

Infection with the RPLPOP2 shRNA lentivirus promotes $R K O$ cell apoptosis. Flow cytometry was also performed to assess apoptosis of RKO cells infected with the RPLPOP2 shRNA lentivirus. The results revealed that apoptosis was significantly increased in RKO cells infected with the RPLPOP2 shRNA lentivirus compared with in the control group (Fig. 6), suggesting that RPLP0P2 inhibits the apoptosis of RKO cells.

\section{Discussion}

With their powerful proliferative ability and low apoptotic rate, CRC cells are strongly resistant to chemotherapy, radiation and other biological treatments, leading to malignant tumor growth and recurrence after resection (21). Previous studies have revealed that lncRNAs are increasingly known to be involved in numerous biological processes, including acceleration of the development and progression of cancer $(22,23)$. It has also been suggested that lncRNA RPLP0P2 levels may be significantly reduced in lung adenocarcinoma (16). Bioinformatics analysis of RPLP0P2 in CRC revealed that RPLP0P2 was negatively associated with overall survival (19), suggesting that RPLP0P2 may serve a key role in CRC. 
A
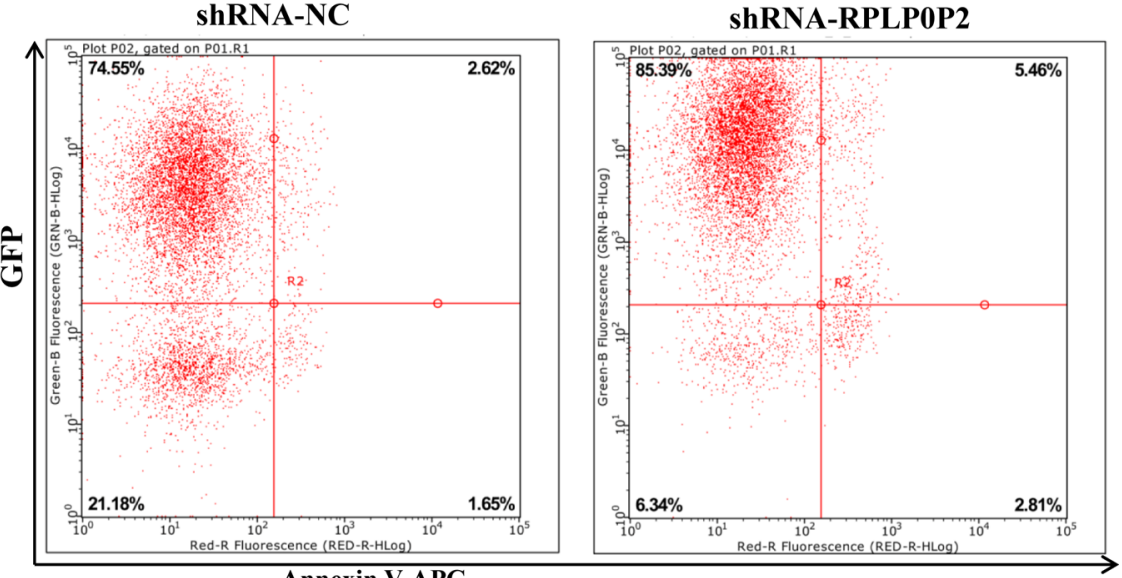

Annexin V-APC
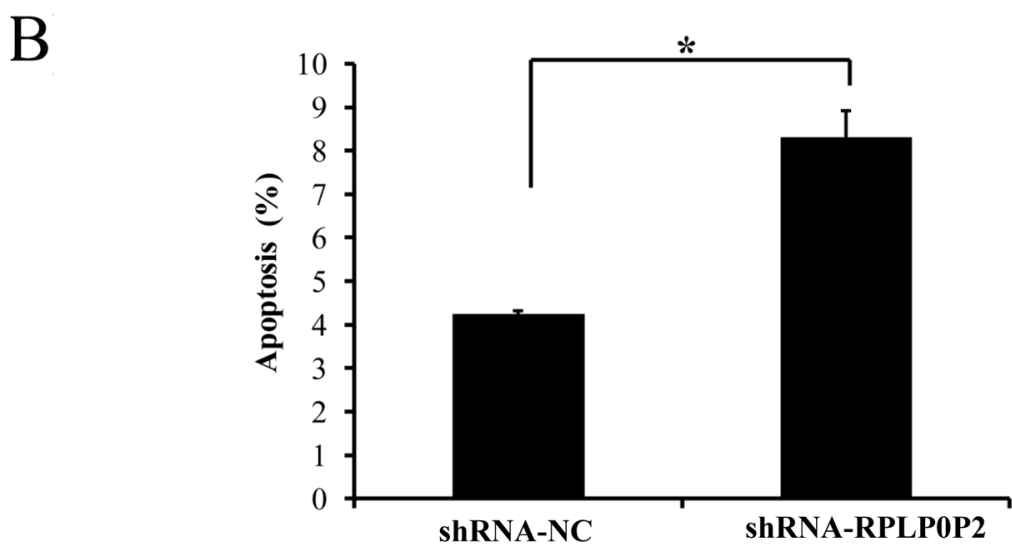

Figure 6. Analysis of apoptosis in lentivirus-infected RKO cells. (A) Apoptosis of RKO cells detected by flow cytometry following infection with the RPLP0P2 shRNA lentivirus. Apoptotic cells are shown in the top right and bottom right quadrants. Y-axis represents the lentivirus carrying enhanced green fluorescent protein transfected cells. X-axis represents APC-stained cells. (B) Apoptotic rate of RKO cells after infection with the RPLP0P2 shRNA lentivirus. * $<0.05$. shRNA, short hairpin RNA; NC, negative control.

The present study performed bioinformatics analysis using publicly available TCGA data. The results revealed that the expression levels of RPLPOP2 were higher in CRC tissues compared with in normal tissues. Further analysis verified that the expression levels of RPLP0P2 were also high in RKO cells. These results indicated that RPLP0P2 may be abnormally expressed in CRC and may act as an oncogene. RPLP0P2 was also previously reported to be negatively associated with overall survival in gastric cancer (18). However, Xu et al (16) reported that RPLP0P2 expression was significantly lower in lung adenocarcinoma compared with in adjacent tissues. These studies indicated that tissue specificity may be one of the most important properties for RPLP0P2.

In the present study, RPLP0P2 knockdown using a shRNA lentivirus suppressed proliferation and colony formation in RKO cells, thus suggesting that RPLPOP2 may be associated with the proliferative capacity of CRC. shRNA-mediated RPLP0P2 knockdown also induced cell cycle arrest of RKO cells in S stage, which confirmed that the RPLP0P2 expression levels were associated with cell proliferation. Chen et al (17) also reported that low expression of lncRNA RPLP0P2 decreased cell proliferation and adhesive ability in lung adenocarcinoma. This finding is in agreement with the present experimental results, which revealed that RPLP0P2 knockdown induced cell accumulation in $\mathrm{S}$ phase of the cell cycle, suggesting that involvement of RPLPOP2 in CRC may be related to cells arrested from $S$ phase to $G_{2} / M$ phase.

The present study demonstrated that RPLP0P2 was associated with the migratory and invasive ability of CRC cells. Furthermore, flow cytometric analysis revealed that RPLP0P2 may inhibit apoptosis of CRC cells, suggesting that RPLP0P2 may promote CRC development via inhibition of apoptosis and metastasis. However, whether and how RPLP0P2 is implicated in CRC requires further study. It has been reported that lncRNA-associated competing endogenous RNA networks, for example, lncRNA-microRNA (miRNA/miR)-mRNA networks, may participate in the progression of CRC (24). Expression of EZH2 has been reported to be associated with the risk of lung cancer (25), and a co-methylation network revealed that RPLP0P2 was co-methylated with EZH2, suggesting involvement of RPLP0P2 in the progression of lung cancer (26). Further analysis revealed that the IncRNA-miRNA-mRNA network miR-29c-3p-RPLP0P2-EZH2 was significantly associated with the prognosis of lung adenocarcinoma (26). These reports may provide further understanding of the role of RPLP0P2 in CRC pathogenesis and prognosis.

In conclusion, the present study revealed that the expression levels of RPLP0P2 were associated with the development of CRC through promotion of proliferation and metastasis, and inhibition of apoptosis. These findings suggested that 
RPLP0P2 may function as an oncogene in CRC, as well as a therapeutic target in CRC treatment.

\section{Acknowledgements}

Not applicable.

\section{Funding}

No funding was received.

\section{Availability of data and materials}

The datasets used and/or analyzed during the current study are available from the corresponding author on reasonable request.

\section{Authors' contributions}

HY, ST and YS conceptualized and designed the study. HY and YM analyzed the data and wrote the manuscript. YM performed the experiments. YS and HY confirmed the authenticity of all the raw data. All authors read and approved the final manuscript.

\section{Ethics approval and consent to participate}

Not applicable.

\section{Patient consent for publication}

Not applicable.

\section{Competing interests}

The authors declare that they have no competing interests.

\section{References}

1. McGuire S: World cancer report 2014. Geneva, Switzerland: World health organization, international agency for research on cancer, WHO Press, 2015. Adv Nutr 7: 418-419, 2016.

2. Gu MJ, Huang QC, Bao CZ, Li YJ, Li XQ, Ye D, Ye ZH, Chen K and Wang JB: Attributable causes of colorectal cancer in China. BMC Cancer 18: 38, 2018.

3. Siegel RL, Miller KD, Fedewa SA, Ahnen DJ, Meester RGS, Barzi A and Jemal A: Colorectal cancer statistics, 2017. CA Cancer J Clin 67: 177-193, 2017.

4. Sana J, Faltejskova P, Svoboda M and Slaby O: Novel classes of non-coding RNAs and cancer. J Transl Med 10: 103, 2012.

5. Gong Z, Zhang S, Zhang W, Huang H, Li Q, Deng H, Ma J, Zhou M, Xiang J, Wu M, et al: Long non-coding RNAs in cancer. Sci China Life Sci 55: 1120-1124, 2012.

6. Wang JP, Tang YY, Fan CM, Guo C, Zhou YH, Li Z, Li XL, Li Y, Li GY, Xiong W, et al: The role of exosomal non-coding RNAs in cancer metastasis. Oncotarget 9: 12487-12502, 2018.

7. Gong Z, Zhang S, Zeng Z, Wu H, Yang Q, Xiong F, Shi L, Yang J, Zhang W, Zhou Y, et al: LOC401317, a p53-regulated long non-coding RNA, inhibits cell proliferation and induces apoptosis in the nasopharyngeal carcinoma cell line HNE2. PLoS One 9: e110674, 2014.

8. Yang L, Tang Y, Xiong F, He Y, Wei F, Zhang S, Guo C, Xiang B, Zhou M, Xie N, et al: LncRNAs regulate cancer metastasis via binding to functional proteins. Oncotarget 9: 1426-1443, 2018.
9. Fan C, Tang Y, Wang J, Xiong F, Guo C, Wang Y, Zhang S, Gong Z, Wei F, Yang L, et al: Role of long non-coding RNAs in glucose metabolism in cancer. Mol Cancer 16: 130, 2017.

10. Gong Z, Yang Q, Zeng Z, Zhang W, Li X, Zu X, Deng H, Chen P, Liao Q, Xiang B, et al: An integrative transcriptomic analysis reveals p53 regulated miRNA, mRNA, and lncRNA networks in nasopharyngeal carcinoma. Tumour Biol 37: 3683-3695, 2016

11. He Y, Jing Y, Wei F, Tang Y, Yang L, Luo J, Yang P, Ni Q, Pang J, Liao Q, et al: Long non-coding RNA PVT1 predicts poor prognosis and induces radioresistance by regulating DNA repair and cell apoptosis in nasopharyngeal carcinoma. Cell Death Dis 9: 235, 2018.

12. Wang Y, Xue D, Li Y, Pan X, Zhang X, Kuang B, Zhou M, Li X, Xiong W, Li G, et al: The long noncoding RNA MALAT-1 is a novel biomarker in various cancers: A meta-analysis based on the GEO database and literature. J Cancer 7: 991-1001, 2016.

13. Zhang W, Huang C, Gong Z, Zhao Y, Tang K, Li X, Fan S, Shi L, Li X, Zhang P, et al: Expression of LINC00312, a long intergenic non-coding RNA, is negatively correlated with tumor size but positively correlated with lymph node metastasis in nasopharyngeal carcinoma. J Mol Histol 44: 545-554, 2013.

14. Zhong Y, Du Y, Yang X, Mo Y, Fan C, Xiong F, Ren D, Ye X, Li C, Wang Y, et al: Circular RNAs function as ceRNAs to regulate and control human cancer progression. Mol Cancer 17: 79, 2018.

15. Zhou R, Wu Y, Wang W, Su W, Liu Y, Wang Y, Fan C, Li X, Li G, Li Y, et al: Circular RNAs (circRNAs) in cancer. Cancer Lett 425: 134-142, 2018

16. Xu G, Chen J, Pan Q, Huang K, Pan J, Zhang W, Chen J, Yu F, Zhou $\mathrm{T}$ and Wang Y: Long noncoding RNA expression profiles of lung adenocarcinoma ascertained by microarray analysis. PLoS One 9: e104044, 2014.

17. Chen J, Hu L, Chen J, Wu F, Hu D, Xu G, Zhu P and Wang Y: Low expression 1ncRNA RPLP0P2 is associated with poor prognosis and decreased cell proliferation and adhesion ability in lung adenocarcinoma. Oncol Rep 36: 1665-1671, 2016.

18. Li CY, Liang GY, Yao WZ, Sui J, Shen X, Zhang YQ, Peng H, Hong WW, Ye YC, Zhang ZY, et al: Integrated analysis of long non-coding RNA competing interactions reveals the potential role in progression of human gastric cancer. Int $\mathrm{J}$ Oncol 48: 1965-1976, 2016.

19. Zhong ME, Chen Y, Zhang G, Xu L, Ge W and Wu B: LncRNA H19 regulates PI3K-Akt signal pathway by functioning as a ceRNA and predicts poor prognosis in colorectal cancer: Integrative analysis of dysregulated ncRNA-associated ceRNA network. Cancer Cell Int 19: 148, 2019.

20. Livak KJ and Schmittgen TD: Analysis of relative gene expression data using real-time quantitative PCR and the 2(-Delta Delta C(T)) method. Methods 25: 402-408, 2001.

21. Selzner M, Bielawska A, Morse MA, Rüdiger HA, Sindram D, Hannun YA and Clavien PA: Induction of apoptotic cell death and prevention of tumor growth by ceramide analogues in metastatic human colon cancer. Cancer Res 61: 1233-1240, 2001.

22. Gupta RA, Shah N, Wang KC, Kim J, Horlings HM, Wong DJ, Tsai MC, Hung T, Argani P, Rinn JL, et al: Long non-coding RNA HOTAIR reprograms chromatin state to promote cancer metastasis. Nature 464: 1071-1076, 2010.

23. Khachane AN and Harrison PM: Mining mammalian transcript data for functional long non-coding RNAs. PLoS One 5: e10316, 2010.

24. Jalali S, Bhartiya D, Lalwani MK, Sivasubbu S and Scaria V: Systematic transcriptome wide analysis of 1ncRNA-miRNA interactions. PLoS One 8: e53823, 2013.

25. Yoon KA, Gil HJ, Han J, Park J and Lee JS: Genetic polymorphisms in the polycomb group gene EZH2 and the risk of lung cancer. J Thorac Oncol 5: 10-16, 2010.

26. Wei Y, Chang Z, Wu C, Zhu Y, Li K and Xu Y: Identification of potential cancer-related pseudogenes in lung adenocarcinoma based on ceRNA hypothesis. Oncotarget 8: 59036-59047, 2017.

This work is licensed under a Creative Commons Attribution-NonCommercial-NoDerivatives 4.0 International (CC BY-NC-ND 4.0) License. 